\title{
Mise Au Point Et Évaluation D'un Système Aquaponique Domestique Dans La Ville Province De Kinshasa (République Démocratique Du Congo)
}

\author{
Cléophas Mikobi Maleshela, Bsc \\ Athanase Kusonika Ndamba, Msc \\ Thierry Tangou Tabou, Phd \\ Shango Mutambwe, Phd
}

Département des Sciences de l'Environnement, Faculté des Sciences, Université de Kinshasa (UNIKIN), Kinshasa XI, R.D Congo.

\section{Crispin Mulaji Kyela, Phd}

Laboratoire de Chimie Analytique et Environnement, Département de Chimie, Faculté des Sciences, Université de Kinshasa (UNIKIN), Kinshasa XI, R.D Congo.

Willy Lusasi Swana, Bsc

Victor Pwema Kiamfu, Phd

Laboratoire de Limnologie, Hydrobiologie et Aquaculture, Département de Biologie, Faculté des Sciences, Université de Kinshasa (UNIKIN), Kinshasa XI, R.D Congo

URL:http://dx.doi.org/10.19044/esj.2020.v16n24p70

\section{Résumé}

L'objectif de cette étude est d'évaluer un système aquaponique mise au point par la réutilisation de l'eau ayant servi à l'élevage des poissons pour produire des légumes. Les paramètres physico-chimiques des eaux du bac d'élevage des poissons, des surverses filtres et d'aquaponie ainsi que le poids (g) et la longueur totale $(\mathrm{mm})$ des poissons Oreochromis niloticus L., 1758 étaient relevés tous les 15 jours. Le diamètre au collet $(\mathrm{cm})$, la hauteur des plants (cm), le nombre des feuilles, le poids moyen (g) des fruits de Allium fistulosom L., 1753, Hibiscus esculentus (L.) M, 1794 et Lactuca sativa L., 1753 cultivés en aquaponie et ceux expérimentés dans les sachets polyéthylène étaient déterminés hebdomadairement. Sur l'ensemble du système aquaponique, la teneur en nitrites a varié de 0,02 à $0,1 \mathrm{mg} / \mathrm{L}$ et celle des nitrates est comprise entre 31 et $48 \mathrm{mg} / \mathrm{L}$. Le $\mathrm{pH}$ de l'eau a fluctué de 6,8 à 7,2. La dureté totale de l'eau a évolué de 3 à $14^{\circ} \mathrm{d}$. Quant à la dureté carbonatée, la teneur a varié de 4 et $7^{\circ} \mathrm{d}$. Pour les plantes cultivées en aquaponie, le diamètre

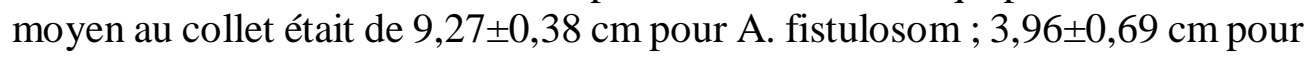


H. esculentus et 5,53 $\pm 0,18 \mathrm{~cm}$ pour L. sativa. Le nombre moyen des feuilles

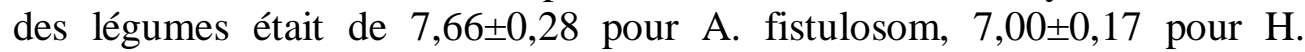

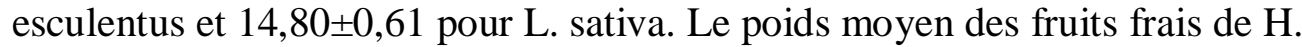
esculentus était de $316,67 \pm 7,1 \mathrm{~g}$. Le taux de survie des poissons était de $72 \pm 3,16 \%$ avec un poids moyen compris entre $2,1 \pm 0,98 \mathrm{~g}$ et $16,17 \pm 4,26 \mathrm{~g}$. Les résultats obtenus sur la stabilité des paramètres physico-chimiques des eaux, la croissance des légumes et poissons expérimentaux ont montré que le système aquaponique semble être un bon milieu de culture capable de contribuer à la croissance des légumes et des poissons mis en culture intégrée.

Mots-clés : Aquaponie, Aquaculture, Agriculture urbaine, Durabilité, Cultures hors-sol, Kinshasa

\title{
Development And Evaluation Of A Domestic Aquaponic System In The City Province Of Kinshasa (Democratic Republic Of Congo
}

\author{
Cleophas Mikobi Maleshela, Bsc \\ Athanase Kusonika Ndamba, Msc \\ Thierry Tangou Tabou, Phd \\ Shango Mutambwe, Phd
}

Département des Sciences de l'Environnement, Faculté des Sciences, Université de Kinshasa (UNIKIN), Kinshasa XI, R.D Congo.

\section{Crispin Mulaji Kyela, Phd}

Laboratoire de Chimie Analytique et Environnement, Département de Chimie, Faculté des Sciences, Université de Kinshasa (UNIKIN), Kinshasa XI, R.D Congo.

\section{Willy Lusasi Swana, Bsc \\ Victor Pwema Kiamfu, Phd}

Laboratoire de Limnologie, Hydrobiologie et Aquaculture, Département de Biologie, Faculté des Sciences, Université de Kinshasa (UNIKIN), Kinshasa XI, R.D Congo

\begin{abstract}
The objective of this study is to evaluate an aquaponics system developed by reusing water from fish farming to produce vegetables. The physico-chemical parameters of the water in the fish rearing tank, filter overflow and aquaponics tanks, as well as the weight (g) and total length (mm)
\end{abstract}


of the fish Oreochromis niloticus L., 1758 were recorded every 15 days. The collar diameter $(\mathrm{cm})$, plant height $(\mathrm{cm})$, number of leaves, average fruit weight (g) of Allium fistulosom L., 1753, Hibiscus esculentus (L.) M, 1794 and Lactuca sativa L., 1753 grown in aquaponics and those tested in polythene bags were determined weekly. Across the entire aquaponic system, nitrite content ranged from 0.02 to $0.1 \mathrm{mg} / \mathrm{L}$ and nitrate content ranged from 31 to $48 \mathrm{mg} / \mathrm{L}$. The $\mathrm{pH}$ of the water ranged from 6.8 to 7.2. Total water hardness varied from 3 to $14^{\circ} \mathrm{d}$. The carbonate hardness varied between 4 and $7^{\circ} \mathrm{d}$. For aquaponically grown plants, the mean diameter at the collar was $9.27 \pm 0.38 \mathrm{~cm}$ for A. fistulosom; $3.96 \pm 0.69 \mathrm{~cm}$ for $\mathrm{H}$. esculentus and $5.53 \pm 0.18 \mathrm{~cm}$ for $\mathrm{L}$. sativa. The mean number of leaves in the vegetables was $7.66 \pm 0.28$ for $\mathrm{A}$. fistulosom, $7.00 \pm 0.17$ for $H$. esculentus and $14.80 \pm 0.61$ for $L$. sativa. The mean weight of fresh fruit for $\mathrm{H}$. esculentus was $316.67 \pm 7.1 \mathrm{~g}$. The survival rate of fish was $72 \pm 3.16 \%$ with an average weight ranging from $2.1 \pm 0.98 \mathrm{~g}$ to $16.17 \pm 4.26 \mathrm{~g}$. The results obtained on the stability of the physico-chemical parameters of the waters, the growth of the experimental vegetables and fish showed that the aquaponic system seems to be a good culture medium capable of contributing to the growth of vegetables and fish in integrated culture.

Keywords: Aquaponia, Aquaculture, Urban Agriculture, Sustainability, Aboveground Crops, Kinshasa

\section{Introduction}

L'agriculture est l'un des domaines les plus importants pour le développement d'un pays, et pour cela la plupart des pays qui ont atteint l'autosuffisance alimentaire ont travaillé dans le développement de ce secteur (Stalport, 2017). L'agriculture conventionnelle ne répond plus aux attentes des citoyens. D'un côté, le cadre législatif de protection de l'environnement se durcit et de l'autre côté, la fertilité des sols pose problème (Bouhenni et Chabani, 2018).

Récemment, le développement de la technologie a rendu possible des avancées majeures dans le domaine de l'agriculture (Alderman, 2015). L'agriculture de précision s'est énormément développée au cours des 20 dernières années afin d'augmenter les rendements et les profits tout en diminuant les impacts environnementaux de l'agriculture traditionnelle (McBratney et al., 2005). Ces rendements sont rendus possibles grâce à la verticalisation des cultures mais aussi grâce à une production non interrompue et un environnement entièrement contrôlé offrant des conditions de croissance optimale, tout en diminuant drastiquement le risque de pertes. Cette idée a connu une énorme propagation dans le monde, et a été adoptée par des populations et même par beaucoup de pays à travers l'industrie. Cette petite idée (avec des grands bénéfices) introduit une nouvelle 
culture dans l'agriculture, celle de concevoir des systèmes fermés mettant les poissons et les plantes l'un au service de l'autre, nommée l'aquaponie (Bouhenni et Chabani, 2018).

En République Démocratique du Congo, l'agriculture reste la principale activité en milieu rural mais, souffre d'une insuffisance voire d'une absence de mécanisation (MINAGRI, 2012). Bien que le pays dispose d'énormes potentialités agricoles, les populations demeurent largement exposées aux risques d'insécurité alimentaire. Les conditions de vie restent très précaires pour le plus grand nombre. Elles se dégradent particulièrement en milieu rural et dans les zones d'insécurité où les populations, souvent en fuite, perdent leurs moyens d'existence. Par ailleurs, l'accroissement démographique de 3,3\% par an (avec 34\% de la population urbaine), les différents régimes fonciers et l'appauvrissement des sols dus à l'exploitation non durable des terres provoquent des tensions souvent exacerbées par des polarisations ethniques qui entretiennent des situations conflictuelles pour l'accès à des terres agricoles (FAO, 2008).

A Kinshasa, pour combler leurs besoins alimentaires en poissons, les kinois font recours la plupart des cas aux produits importés (Lusasi et al., 2019a ; 2019b). Ceci a créé une dépendance de cette ville province voir du pays vis-à-vis de l'extérieur et constitue un véritable manque à gagner pour l'économie nationale. Mais, malgré le volume croissant des importations des produits alimentaires, la consommation en calories est inférieure à celle des bilans d'approvisionnement alimentaire : 1,368 calories et 38,5g de protéines/personne/jour en 2015 au lieu de 2,300 calories et de 70 g de protéines/personne/jour (Tollens, 2015).

Kinshasa est une ville dotée d'un réseau hydrographique riche en ressources halieutiques mais, exploiter de manière artisanale (Lusasi et al., 2019b). La ville comprend des vastes étendues des zones humides et des terres agricoles qui, furent, il y a quelques dizaines d'années le grenier de la ville.

Face à cette situation qui prévaut à Kinshasa, des solutions durables doivent être trouvées. Ainsi, le développement de l'aquaponie urbaine pourrait contribuer à répondre aux besoins alimentaires de la population, au développement socio-économique de la ville et à la conservation des ressources halieutiques du pays.

La présente étude a pour objectif d'évaluer le rendement des légumes et des poissons dans un système aquaponique mise au point dans un laboratoire expérimental installé dans la ville province de Kinshasa en République Démocratique du Congo. 


\section{Matériel et méthodes}

\subsection{Cadre d'étude}

Les expériences se sont déroulées dans un laboratoire expérimental installé dans la ville province de Kinshasa en République Démocratique du Congo, plus précisément dans la commune de Ngaliema au quartier Binza-Pigéon.

\subsection{Méthodologie}

\subsubsection{Matériel biologique}

Le matériel biologique utilisé dans cette étude est composé des légumes (Allium fistulosom L., 1753, Hibiscus esculentus (L.) M., 1794 et Lactuca sativa L., 1753) et des poissons (Oreochromis niloticus L., 1758).

\subsubsection{Choix des légumes et des poissons expérimentaux}

Le choix du matériel végétal (Allium fistulosom: la courge, Hibiscus esculentus : le gombo et Lactuca sativa : la laitue) est basé sur le fait que ces légumes sont parmi ceux qui ont une bonne croissance dans le système aquaponique (CRAAQ, 2012) et font aussi partie de ceux qui sont les plus rencontrés sur les marchés de Kinshasa. La laitue par exemple, a toujours été une excellente candidate pour la production hydroponique. Sa culture est maitrisée et facile à intégrer dans un plan économique viable. De plus, celleci s'adapte à des conditions plus défavorables, dont celle des eaux plus pauvres en nutriments. Ce qui fait d'elle une culture phare de l'aquaponie (Kloas, 2015).

Les poissons sont la centrale électrique d'un système aquaponique qui fournit les nutriments pour les plantes. Si les poissons sont comestibles, ils fournissent également des protéines d'origine animale pour l'homme. Garder les poissons dans un système aquaponique est plus simple que de garder les poissons dans un aquarium. En suivant correctement les directives de suivi d'un système aquaponique, la croissance des poissons de la taille des alevins aux poissons prêts à manger peut être extrêmement simple (Laviale, 2015).

Le choix porté sur Oreochromis niloticus se justifie dans le sens où, c'est l'une des meilleures espèces pour l'aquaculture africaine en générale et congolaise en particulière (Lusasi et al., 2019a). Bien connue et parfaitement maîtrisée, elle est capable de fabriquer des protéines de qualité à partir des sousproduits agro-industriels de faible valeur nutritive. Cette espèce de poisson bat également le record de croissance parmi les poissons élevés (Micha et al., 1975 ; Mélard et Philippart, 1981 ; Kestemont et al., 1989 ; Lazard, 2009) en Afrique en général et plus particulièrement en R.D Congo. Ce poisson est parfaitement intégré dans l'alimentation des congolais. Il se prête à des combinaisons culinaires variées depuis la préparation du poisson frais, salé ou fumé mis dans la sauce jusqu'au poisson préparé à l'étouffé. Lusasi et al., 
(2019b) ; Masua et al., (2020) soutiennent qu'O. niloticus fait partie des poissons les plus vendus dans les marchés de Kinshasa en R.D Congo.

\subsubsection{Mise en place des pépinières}

Trois simples et petites pépinières de $3 \times 1 \mathrm{~m}$ ont été établis afin d'assurer un approvisionnement permanent de l'unité aquaponique en légumes sains et prêts à être cultivés dans le système aquaponique. Les semences étaient achetées au Centre de Production des Semences (CEPROSEM) situé dans la commune de Mont-Ngafula à Kinshasa. Dans les pépinières, les plantes étaient retirées 7 jours après pour être placées dans le système hydroponique ainsi que dans les sachets en polyéthylènes pour les lots tests.

\subsubsection{Structure expérimentale du système aquaponique}

Le dispositif expérimental (figure 1) était constitué d'une cuve IBC de capacité de 1000 litres-pour l'élevage des poissons, d'un fût en plastique de 20 litres utilisé comme filtre contenant les mousses pour retenir les déchets solides (aliments non consommés, excréments...) et les bouchons des bouteilles en plastiques pour servir de support aux bactéries (AQUAVET, 2014). Quatre bassins de culture des légumes portaient les tasseaux contenant les plantes aux racines supportées par les billes d'argile et le tout porté par quatre radeaux de 15 trous ou alvéoles surnageant dans l'eau qui provient du filtre. Une motopompe de marque OASE remontait l'eau de la cuve IBC vers le filtre. Un aérateur de marque Aqua Forte était installé dans le bac d'élevage des poissons.
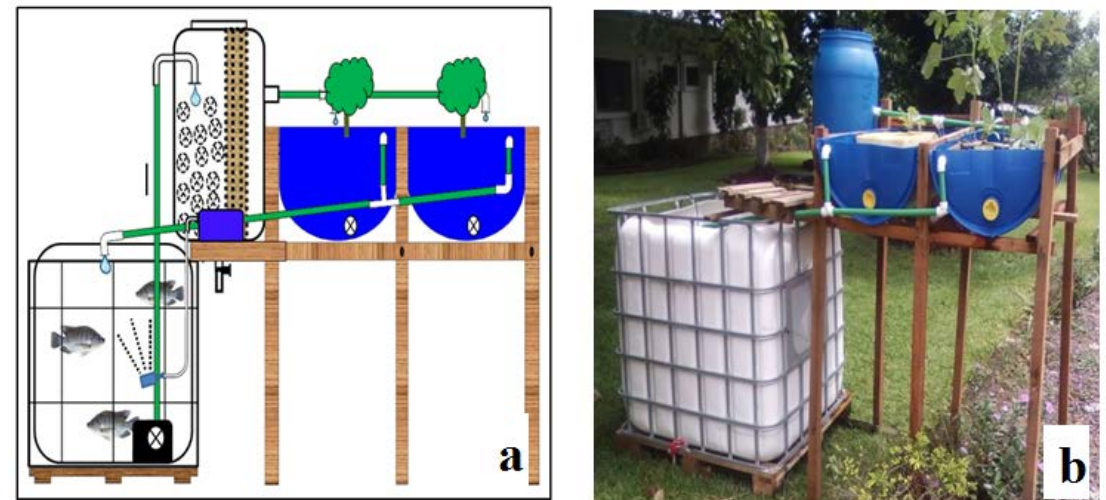

Figure 1 : Design du système aquaponique (a) et système aquaponique mis au point (b)

\subsubsection{Cultures des légumes et mise en charge des poissons dans I e système aquaponique}

Le transfert des jeunes plants de la pépinière aux rigoles de culture aquaponique a été fait tout en évitant au maximum les chocs mécaniques (ou stress) de ces derniers. Chaque plante était retirée soigneusement de la pépinière et ses racines nettoyées avec de l'eau de robinet avant d'être placés dans un tasseau en plastique. L'espacement des tasseaux sur le radeau était de 20 sur $20 \mathrm{~cm}$ pour la laitue et 30 sur $30 \mathrm{~cm}$ pour la courge et le gombo. Afin 
de comparer la croissance des plantes cultivées dans le système aquaponique et celles expérimentées dans le sol ferme, des semis ont été réalisés dans des sachets en polyéthylènes contenant le sol jusqu'au 2/3.

En ce qui concerne les poissons, cent cinquante (150) alevins des poissons Oreochromis niloticus L., 1758 (poids moyen initial de 2,1 $\pm 0,98$ g et 28,1 $\pm 0,37 \mathrm{~mm}$ de longueur totale moyenne initiale) ont été achetés au mois d'août 2017 à la ferme Mboma, située dans la commune de la N'sele à Kinshasa. Ces derniers étaient transportés dans des sachets plastiques de $10 \mathrm{~kg}$ oxygénés au 2/3 du sachet avant d'être emballés. Les poissons étaient placés dans une cuve IBC contenant 800 litres d'eau pour leur élevage dans le système aquaponique.

\subsubsection{Suivi de la qualité des eaux du système aquaponique}

Les paramètres physico-chimiques $(\mathrm{pH}$, dureté totale $(\mathrm{GH})$, nitrites, nitrates et stabilité du $\mathrm{pH}$ de l'eau $(\mathrm{KH})$ ) des eaux de la cuve d'élevage des poissons, des surverses filtre et d'aquaponie étaient mesurés à l'aide d'un kit de test de type Easy Test 6 in 1 et la sonde multiparamétriques Combo de marque HANNA. Le prélèvement de ces paramètres avait lieu tous les 15 jours et pendant 90 jours.

\subsubsection{Evaluation de la croissance des légumes}

Le diamètre au collet $(\mathrm{cm})$, la hauteur des plants $(\mathrm{cm})$, le nombre des feuilles, le poids ( $\mathrm{g}$ ) des fruits des plantes cultivées en aquaponie et celles cultivées dans des sachets polyéthylène étaient estimés hebdomadairement. Le relevé des différents paramètres liés à la croissance des légumes était rendu possible grâce à un pied à coulisse électronique (précision $0,1 \mathrm{~mm}$ ), un mètre ruban de $200 \mathrm{~cm}$ ainsi qu'une balance électronique de marque Salter (précision $0,1 \mathrm{~g})$.

\subsubsection{Préparation de l'aliment des poissons}

L'a distribué aux poissons a été fabriqué avec des sous-produits agricoles loc aux (déchets de manioc, son de riz et de blé, tourteau d'arachide, tourteau de s oya, farine de poisson et de sang, coquilles d'œufs) en y ajoutant de l'huile d e palme et du sel de cuisine. Les ingrédients utilisés ont été choisis en fonctio n de leur valeur nutritive, leur disponibilité sur le marché de Kinshasa et de le ur coût selon les suggestions faites par Lusasi et al., (2019a). La composition bromatologique de l'aliment élaboré et distribué aux alevins des poissons $O r$ eochromis niloticus est présentée dans le tableau 1. L'aliment a été analysé a u laboratoire de nutrition et diététique/ISTM Kinshasa.

Tableau 1 : Composition bromatologique de l'aliment expérimental

\begin{tabular}{ccc}
\hline Composition & Matière sèche & Matière fraiche \\
\hline Humidité (\%) & - & $12,2 \pm 0,34$ \\
Protéines (\%) & $34,98 \pm 0,084$ & $30,71 \pm 0,18$ \\
Lipides (\%) & $14,8 \pm 0,72$ & $12,99 \pm 0,23$ \\
Cendres (\%) & $8,2 \pm 0,34$ & $7,19 \pm 0,29$ \\
Fibres (\%) & $9,73 \pm 0,24$ & $8,54 \pm 0,38$
\end{tabular}




$\begin{array}{ccc}\text { Glucides (\%) } & 32,29 \pm 0,34 & 28,35 \pm 0,25 \\ \text { Energie (Kcal) } & 396,28 & 353,09\end{array}$

\subsubsection{Nourrissage des poissons}

Les poissons étaient nourris à la main libre deux fois par jour (9 h00' et 15h00'). La quantité journalière d'aliment distribué aux poissons correspondait à $10 \%$ de leur biomasse totale conformément aux propositions faites par Lusasi et al., (2019a). Après chaque pêche de contrôle, la quantité d'aliment à distribuer les jours suivants, était ajustée par rapport à l'évolution du poids moyen des poissons.

\subsubsection{Pesée et mensuration des poissons}

Les poissons étaient pesés (g) à l'aide d'une balance électronique de marque Salter (précision : $0,1 \mathrm{~g}$ ) et étaient mesurés (au mm près) avec un ichtyomètre électronique tous les 15 jours à partir du jour de la mise en charge des alevins.

\subsubsection{Evaluation des paramètres zootechniques des poissons}

Les paramètres zootechniques et indices piscicoles ci-après ont été évalués pour caractériser la croissance des alevins des poissons Oreochromis niloticus L., 1758 (Fiogbe et al., 2009 ; Lusasi et al., 2019a) :

- $\mathrm{TS}=(\mathrm{Nf} / \mathrm{Ni}) \times 100$

TS (\%) : Taux de survie, Nf: Nombre final des poissons et Ni : Nombre initial des poissons.

- $\quad \mathrm{PM}=\mathrm{B} / \mathrm{Np}$

PM : Poids Moyen des poissons (g), B : Biomasse (g) et Np : Nombre des poissons.

- $\mathrm{TM}: \mathrm{TGP} / \mathrm{Np}$

TM : Taille Globale des Poissons (mm) et Np : Nombre des poissons.

- $\quad \mathrm{GPM}=$ Pmf-Pmi

GPM : Gain de Poids Moyen, Pmf : Poids moyen final des poissons

(g) et Pmi : Poids moyen initial des poissons (g).

\subsubsection{Analyse et traitement statistiques des données}

Les données obtenues sur la variation des différents paramètres physicochimiques des eaux, la croissance des légumes expérimentaux et les données des paramètres zootechniques des poissons ont été encodées sur le tableur Excel 2013 avant de les soumettre aux analyses statistiques. La comparaison des paramètres phytotechniques des légumes a été faite en appliquant le test du Tukey HSD. Le test de Student a été appliqué à l'intervalle de 95\% de confiance aux données liées à la croissance des poissons. Le logiciel Origin 6.1 a été utilisé à cet effet. 


\section{Résultats}

\subsection{Paramètres physico-chimiques des eaux}

La variation des différents paramètres physico-chimiques des eaux utilisées dans le bac d'élevage des poissons, des surverses filtre et hydroponique du début jusqu'à la fin de l'expérience est consignée dans le tableau 2. Au début de l'expérience, la teneur en $\mathrm{NO}_{2}$ dans le bac des poissons est de $0,08 \mathrm{mg} / \mathrm{L}$ et $0,01 \mathrm{mg} / \mathrm{L}$ à la fin. Dans la surverse filtre cette concentration est de $0,1 \mathrm{mg} / \mathrm{L}$ au début et $0,02 \mathrm{mg} / \mathrm{L}$ à la fin de l'expérimentation tandis qu'elle est de 0,06 $\mathrm{mg} / \mathrm{L}$ au début dans la surverse d'hydroponie et $0,01 \mathrm{mg} / \mathrm{L}$ à la fin de l'expérience. La concentration en $\mathrm{NO}_{3}$ est de $0,7 \mathrm{mg} / \mathrm{L}$ au début de l'étude et de $0,4 \mathrm{mg} / \mathrm{L}$ à la fin dans le bac des poissons. Dans la surverse filtre, la concentration est de $10 \mathrm{mg} / \mathrm{L}$ au début de l'expérience et $48 \mathrm{mg} / \mathrm{L}$ à la fin de l'expérience. L'eau de la surverse hydroponique a présenté la teneur en $\mathrm{NO}_{3}$ comprise entre 0,9 et $0,8 \mathrm{mg} / \mathrm{L}$ du début jusqu'à la fin de l'expérience. Le potentiel hydrogène au début de l'expérience est de 7,4; 7,5 et 7,6 respectivement dans le bac des poissons ainsi que dans les surverses filtre et hydroponique. A la fin de l'expérience, les trois traitements présentent la même valeur de $\mathrm{pH}$ (soit 6,8). La dureté totale des eaux la plus élevée est enregistrée dans la surverse filtre $\left(4^{\circ} \mathrm{d}\right.$ au début de l'expérience et $14^{\circ} \mathrm{d}$ à la fin) ensuite dans la surverse hydroponique $\left(4^{\circ} \mathrm{d}\right.$ au début de l'expérience et $10^{\circ} \mathrm{d}$ à la fin) et enfin la faible teneur est obtenue dans le bac des poissons $\left(3^{\circ} \mathrm{d}\right.$ au début de l'expérience et 4 à la fin). La dureté carbonatée enregistrée au début de l'expérience dans le bac des poissons ainsi que dans les surverses filtre et hydroponique est de $4^{\circ} \mathrm{d}$ respectivement. A la fin de l'expérience, la teneur de $6^{\circ} \mathrm{d}$ a été obtenue dans les trois traitements.

Tableau 2 : Variation des paramètres physico-chimiques

\begin{tabular}{|c|c|c|c|c|c|c|c|c|c|c|}
\hline \multirow{3}{*}{ Traitements } & \multicolumn{10}{|c|}{ Paramètres physico-chimiques des eaux } \\
\hline & \multicolumn{2}{|c|}{$\mathrm{NO}_{2}(\mathrm{mg} / \mathrm{L})$} & \multicolumn{2}{|c|}{$\mathrm{NO}_{3}(\mathrm{mg} / \mathrm{L})$} & \multicolumn{2}{|c|}{ pH } & \multicolumn{2}{|c|}{ GH $\left({ }^{\circ} \mathbf{d}\right)$} & \multicolumn{2}{|c|}{$\mathbf{K H}\left({ }^{\circ} \mathbf{d}\right)$} \\
\hline & D.E & F.E & D.E & F.E & D.E & F.E & D.E & F.E & D.E & F.E \\
\hline Bac des po & 0,08 & 0,01 & 0,7 & 0,4 & 7,4 & 6,8 & 3 & 4 & 6 & 4 \\
\hline Surverse filtre & 0,1 & 0,02 & 10 & 48 & 7,5 & 6,8 & 4 & 14 & 6 & 4 \\
\hline Surverse hydroponique & 0,06 & 0,01 & 0,9 & 0,8 & 7,6 & 6,8 & 4 & 10 & 6 & 4 \\
\hline
\end{tabular}

D.E = Début Expérience ; F.E = Fin Expérience 


\subsection{Productivité végétale}

Les données moyennes relatives à la croissance des légumes cultivés en aquaponie et ceux expérimentés dans des sachets en polyéthylène à la fin de l'expérimentation sont consignées dans le tableau 3.

Tableau 3 : Valeurs moyennes des paramètres de croissance des légumes cultivés en aquaponie et en sachets en polyéthylène à la fin de l’expérimentation (nombre des spécimens des plantules égale à 9 pour chaque espèce et - : absence).

\begin{tabular}{|c|c|c|c|c|c|c|}
\hline \multirow{3}{*}{ Paramètres de croissance } & \multicolumn{6}{|c|}{ Milieu de culture } \\
\hline & \multicolumn{3}{|c|}{ Aquaponie } & \multicolumn{3}{|c|}{ Sachets } \\
\hline & Allium fistulosom & $\begin{array}{l}\text { Hibiscus } \\
\text { esculentus }\end{array}$ & Lactuca sativa & $\begin{array}{c}\text { A. } \\
\text { fistulosom }\end{array}$ & H. esculentus & L. sativa \\
\hline Diamètre au collet (cm) & $9,27 \pm 0,38$ & $3,96 \pm 0,69$ & $5,53 \pm 0,18$ & $7,00 \pm 0,57$ & $2,33 \pm 0,97$ & $2,04 \pm 0,86$ \\
\hline Hauteur du plant $(\mathrm{cm})$ & $2,12 \pm 0,46$ & $2,32 \pm 0,13$ & - & $1,63 \pm 0,83$ & $2,21 \pm 0,11$ & 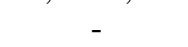 \\
\hline Nombre des feuilles & $7,66 \pm 0,28$ & $7,00 \pm 0,17$ & $14,80 \pm 0,61$ & $4,33 \pm 0,16$ & $3,33 \pm 0,63$ & $20,33 \pm 0,32$ \\
\hline Poids des feuilles fraiches (g) & $316,67 \pm 8,04$ & - & $330,66 \pm 12,1$ & $240,00 \pm 11,1$ & - & $217,55 \pm 9,01$ \\
\hline Poids moyen des fruits frais (g) & - & $316,67 \pm 7,1$ & - & - & $240,00 \pm 11,22$ & - \\
\hline $\mathrm{F}$ & 70,71 & 30,25 & 39,52 & 3,99 & 6,45 & 20,51 \\
\hline P Value & 0,001 & $<0,001$ & $<0,001$ & 0,116 & 0,064 & 0,010 \\
\hline
\end{tabular}

Les résultats des différents paramètres phytotechniques résumés dans le tableau 3 ci-dessus et leur comparaison à l'aide de la méthode de Tukey HSD laissent voir que les légumes expérimentés en aquaponie (figure 2) présentent une meilleure croissance ( $\mathrm{F}=70,71 ; \mathrm{p}=0,001$ pour Allium fistulosom, $\mathrm{F}=30,25 ; \mathrm{p}<0,001$ pour Hibiscus esculentus et $\mathrm{F}=39,52 ; \mathrm{p}<$ 0,001 pour Lactuca sativa) que ceux qui sont expérimentés dans les sachets en polyéthylène. 


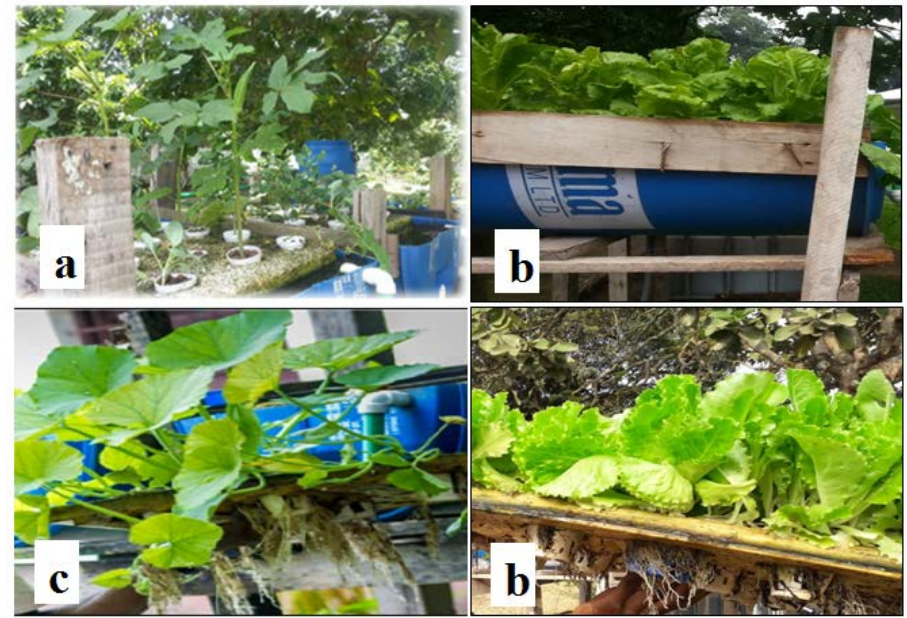

Figure 2 : Production des légumes dans le système aquaponique (a) : gombo, (b) : laitue et

\subsection{Indices piscicoles}

(c) : courge

Les paramètres zootechniques relatifs à l'élevage des poissons Oreochromis niloticus L., 1758 dans le système aquaponique ont été déterminés de la manière suivante :

\subsubsection{Taux de survie}

Le nombre des poissons est passé de 150 au début de l'expérimentation à 109 à la fin de l'expérience, soit un taux de survie de 72ะ3,16\% (figure 3). La période allant du $75^{\text {ème }}$ au $90^{\text {ème }}$ jours est celle qui a enregistré plus des cas de mortalité tandis que la période allant du $15^{\text {ème }}$ au $30^{\text {ème }}$ jours n’a pas connu des cas de mortalité.

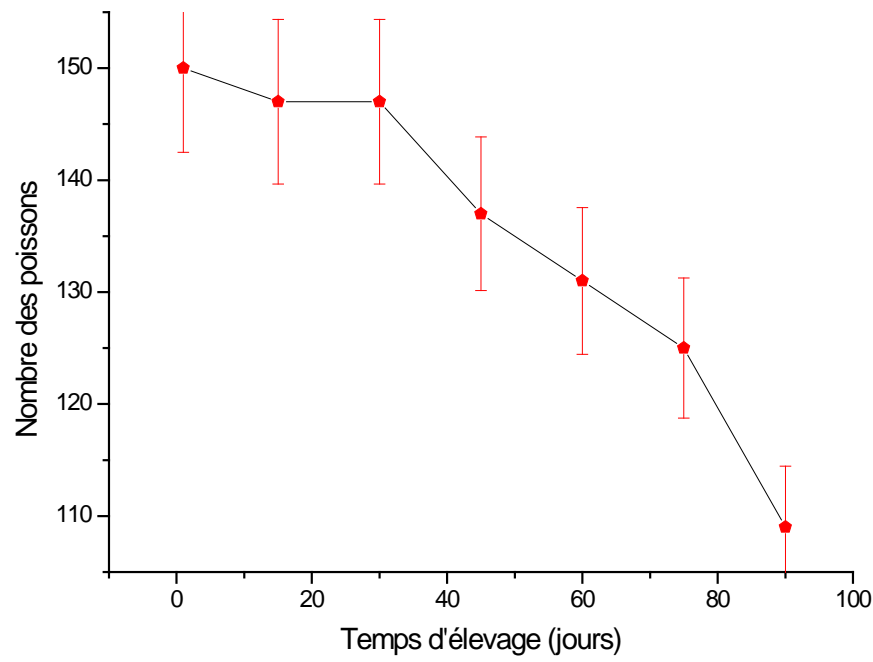

Figure 3 : Variation du taux de survie des poissons (\%) en fonction du temps d'élevage 


\subsubsection{Poids moyen et taille moyenne des poissons expérimentés}

Le poids moyen $(\mathrm{g})$ des poissons élevés a évolué d'une période à une autre.

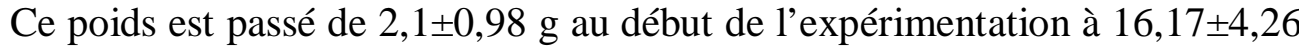
$\mathrm{g}$ à la fin de l'essai. Le test statistique de Student appliqué aux données liées à la croissance pondérale des poissons montre que la différence de croissance est hautement significative $(t=4,224 ; p=0,005)$. La meilleure croissance pondérale est observée entre les $75^{\text {ème }}$ et $90^{\text {ème }}$ jours $(11,66 \pm 0,31 \mathrm{~g}$ à $16,17 \pm 1,21 \mathrm{~g})$. La valeur la plus faible est enregistrée entre le $1^{\text {er }}$ et les $15^{\text {ème }}$ jours $(2,1 \pm 0,98 \mathrm{~g}$ à $3,45 \pm 0,12 \mathrm{~g})$. La taille moyenne $(\mathrm{mm})$ des poissons est

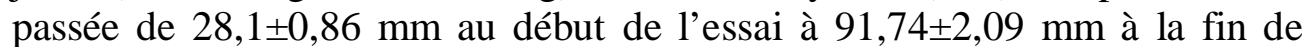
l'expérience. Le test de Student appliqué aux données liées à la croissance en longueur des alevins révèle une différence très significative $(t=6,442 ; \mathrm{p}=$ 0,018) entre les moyennes. La meilleure croissance linéaire est observée entre

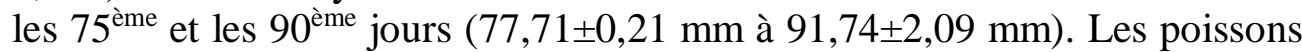
ont enregistré une faible croissance $(28,1 \pm 0,86 \mathrm{~mm}$ à $34,07 \pm 0,48 \mathrm{~mm})$ durant la période allant du $1^{\text {er }}$ au $15^{\text {ème }}$ jours (figure 4)
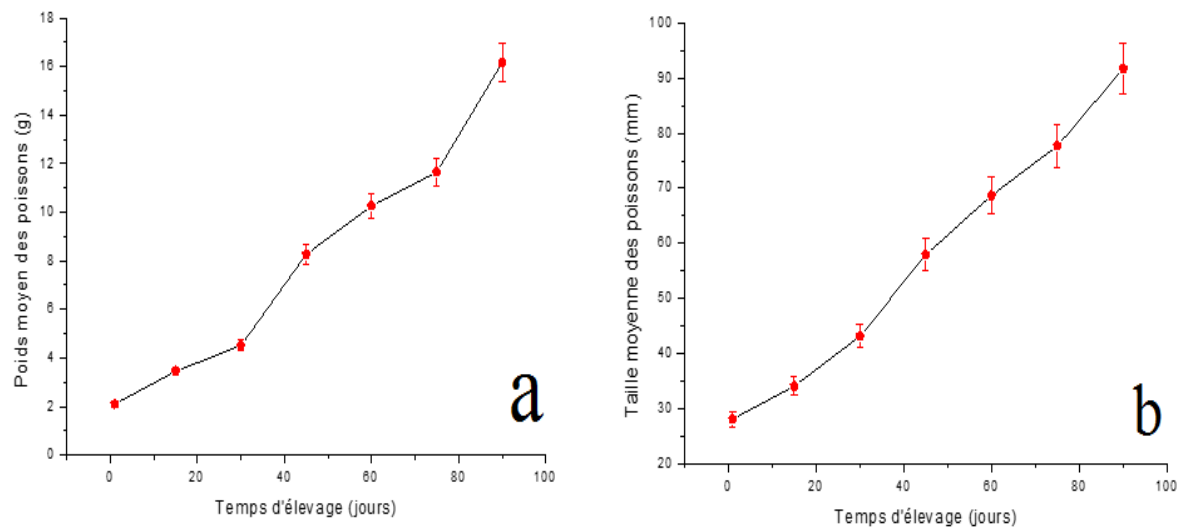

Figures 4 : Evolution du poids moyen (a) et taille moyenne (b) des poissons en fonction du temps d'élevage

\subsubsection{Gain de poids moyen des poissons}

L'analyse statistique des données relatives au gain de poids moyen des poissons par le test de Student a démontré qu'il existe une différence très significative $(\mathrm{t}=8,15$ et $\mathrm{p}=0,019)$ entre les moyennes d'une période à l'autre. Il ressort que le gain de poids le plus élevé des poissons est obtenu entre le $45^{\text {ème }}$ et le $60^{\text {ème }}$ jours $(4,51 \mathrm{~g})$. Le plus faible est relevé entre le $1^{\text {er }}$ et le $14^{\text {ème }}$ jours (2,07 g) (figure 5). 


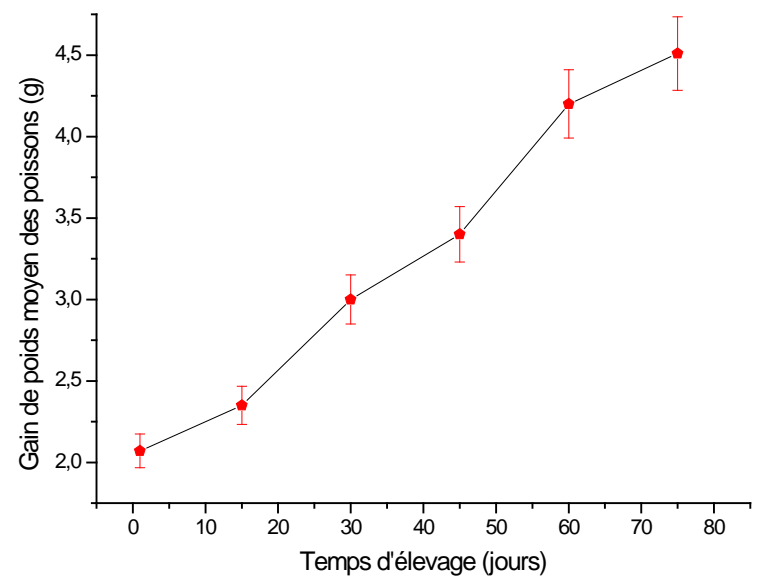

Figure 5 : Gain de poids moyen des poissons (g) en fonction du temps d’élevage

\section{Discussion}

Après analyse et traitement des différentes données, les résultats obtenus sur les paramètres physico-chimiques des eaux ont montré que la teneur en nitrites était élevée au début de l'expérience et a diminué progressivement à la fin de l'expérimentation. Celle des nitrates était faible au début de l'expérimentation et a augmenté progressivement jusqu'à la fin de l'expérience. Ces valeurs élevées seraient dues à la dégradation des résidus d'aliments non consommés par les poissons et de l'accumulation des déjections des poissons dans l'eau conformément aux observations faites par Saidani (2016). D’après ce dernier, les différences de concentration en nitrites et nitrates correspondent à la phase d'installation, la multiplication et la formation des colonies des bactéries nitrifiantes (Nitrosomonas et Nitrobacter). Ces observations indiquent aussi que ces bactéries convertissent l'ammoniac en nitrite puis en nitrate (MDDELCC, 2017). Les concentrations en nitrites obtenues dans cette étude sont en dessous de la valeur de seuil de tolérance $(\leq 1,0 \mathrm{mg} / \mathrm{L})$ et celles de nitrates sont proches de la norme recommandée $(\leq 50 \mathrm{mg} / \mathrm{L})$ pour la survie des poissons Oreochromis niloticus en élevage (FAO, 2017). De ce fait, les teneurs en nitrites et nitrates observées au cours de cette expérimentation ne peuvent pas occasionner des effets néfastes sur la croissance des poissons et des légumes expérimentaux suivant les observations et recommandations faites par Bouhenni et Chabani (2018) dans une étude sur la réalisation d'une micro-ferme aquaponique et développement d'une application de gestion commerciale pour les projets d'aquaponie en Algérie. Heliott (2014) fait savoir que dans l'environnement, les concentrations en nitrites et nitrates sont très rarement élevées dans les milieux aquatiques moins pollués (rarement supérieur à $0,5 \mathrm{mg} / \mathrm{L}$ pour les nitrites et $50 \mathrm{mg} / \mathrm{L}$ pour les nitrates). C'est le cas de nos travaux. 
Le $\mathrm{pH}$ de l'eau a varié entre 6,8 et 7,2 du début jusqu’à la fin de l'expérimentation. Ces valeurs sont restées dans la fourchette qui corresponde à l’optimum (7 et 7,2) pour les besoins des bactéries du filtre biologique (FAO, 2017). Par contre, ces résultats sont en contradiction avec ceux obtenus par Laviale (2015) lors d’une expérimentation et démonstration aquaponique à vocation pédagogique en France. Les résultats obtenus par cet auteur montrent une chute du pH (5,6 à 4,3) du début jusqu'à la fin de l'expérimentation. D’après Kestemont et al,. (1989) ; Pouomogne (1998) ; Munganga et al., (2020), les poissons et les plantes aquatiques évoluent bien dans un pH compris entre 6 et 8 . Les mêmes observations se rapprochent de celles faites par Foucard et al., (2015) dans une étude consacrée sur les productions piscicoles et horticoles dans un système aquaponique en France.

Concernant la productivité végétale, il n’y a pas eu des changements significatifs dans la croissance des plantes expérimentées dans le système aquaponique au cours de la première semaine. Ceci s'explique par le fait qu'au début de tout expérience en aquaponie, il y a un manque d'ammoniac produit par les poissons d'après le constat fait par Piccolo et al., (2013) ; Bouhenni et Chabani (2018) lors des expériences similaires à celle-ci. A la fin de l'essai, les légumes cultivés dans le système aquaponique ont donnés un meilleur rendement que ceux qui ont été cultivé dans les sachets en polyéthylène. Le poids moyen des fruits frais d'Hibiscus esculentus était de 316,67士7,1 g pour

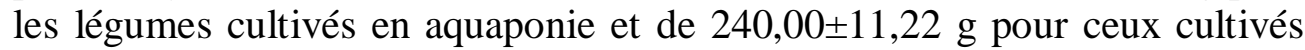
dans des sachets en polyéthylène. Ces résultats sont similaires à ceux de Gidinda (2016) qui soutient que pour les cultures au sol, des tels rendements sont dû aux conditions édaphiques défavorables en début de croissance du Gombo. Les mêmes observations ont été également faites par Diansongi (2018) qui, a obtenu une moyenne de diamètre au collet de 2,98 $\pm 0,19 \mathrm{~cm}$ et une moyenne des 14,24 $\pm 2,41$ feuilles pour la laitue.

L'analyse de la croissance des poissons expérimentaux a montré que le taux

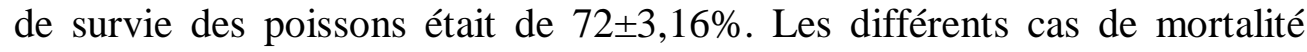
étaient relevés souvent après les pêches de contrôle. Cela serait dû probablement aux stress des manipulations des poissons conformément aux observations faites par Lusasi et al., (2019a). Les poissons ont présenté une bonne croissance pondérale et linéaire du début jusqu'à la fin de l'expérience. La croissance des poissons a bien évolué à la seconde période d'essai en présentant des moyennes supérieures à la première période d’expérimentation. Ces observations se rapprochent de celles faites par Adjanke (2011) lors de la production d'alevins et la gestion d'une ferme piscicole au Togo d'une durée d’élevage de 95 jours. 


\section{Conclusion}

Cette étude avait pour objectif de mettre au point et d'évaluer un système aquaponique domestique pour produire les légumes et les poissons pouvant contribuer à la lutte contre l'insécurité alimentaire et la pauvreté qui sévit à Kinshasa. Les teneurs des différents paramètres physico-chimiques $(\mathrm{pH}$, dureté totale (GH), nitrites, nitrates et stabilité du $\mathrm{pH}(\mathrm{KH})$ ) des eaux utilisées pour la co-culture des légumes et des poissons au cours de cette expérience sont restées dans le seuil de tolérance exigé pour faciliter la croissance des légumes et des poissons expérimentaux. Les légumes expérimentés dans le système aquaponique ont montré une meilleure croissance que ceux expérimentés dans les sachets en polyéthylène. La stabilité des paramètres physico-chimiques des eaux, la bonne productivité végétale observée en aquaponie ainsi que la croissance des poissons expérimentés montrent que le système aquaponique est un bon milieu de culture capable de faciliter la croissance des légumes et des poissons cultivés ensemble avec un bon rendement à la récolte.

\section{Remerciements}

Les auteurs remercient tous les techniciens de la Ferme et Ecloserie de La Kinoise des Poissons pour leur apport technique. Merci à Messieurs Norman KAMOSI et Yves MUTOMBO pour les matériels fournis.

\section{References:}

1. Adjanke, A. (2011). Production d'alevins et Gestion de ferme piscicole. Formation en pisciculture. Coordination Togolaise des Organisations Paysannes et de Producteurs Agricoles, 39 p.

2. Alderman, S. (2015). The pratically and sustainablity of aquaponic agriculture versus traditional agriculture with emphasis on application in the middle. San Marcos, 46 p.

3. AQUAVET (2014). Description du système aquaponique : résultats $\mathrm{n}^{\circ} 2.21 \mathrm{p}$.

4. Bouhenni, K. I. et Chabani, R. (2018). Réalisation d'une micro-ferme aquaponique et développement d'une application de gestion commerciale pour les projets d'aquaponie. Mémoire de Master en Génie industriel, spécialité ingénierie de la production. Université Abou bekr Belkaid-Tlemcen, Faculté de Technologie, Algérie, 101 p.

5. CRAAQ (Centre de Référence en Agriculture et Agroalimentaire du Québec). (2012). Références économiques. Tomates, concombres et laitues en serre. Budget d'entreprise, Agriculture, Pêcheries et Alimentation, Québec, 9 p.

6. Diansongi, K. (2018). Evaluation de l'effet résiduel de Mycorhizes (Funneli formismossae) sur la production de la laitue dans les 
conditions Agro-écologiques de Kinshasa/Mont-Amba. Travail de fin de cycle, Faculté des Sciences Agronomiques, Université de Kinshasa, R.D Congo, $40 \mathrm{p}$.

7. FAO. (2008). Document d'introduction aux concepts de la sécurité alimentaire. Programme CE-FAO « Sécurité alimentaire l'information pour l'action », $38 \mathrm{p}$.

8. FAO. (2017). Cultured aquatic species fact sheets Oreochromis niloticus

(Linnaeus,

1758).

http://www.fao.org/fishery/culturedspecies/Oreochromis_niloticus/fr. Consulté en ligne le 20 juin 2019.

9. Fiogbe, E. D., Akitikpa, B. et Accodji, J-.M. M. (2009). Essais de mise au point de formules alimentaires à base d'azolla (Azolla microphylla Kaulf) et de sous-produits locaux pour la pisciculture rurale du Tilapia Oreochromis niloticus L. International Journal of Biological and Chemical Sciences, 3(2): 398-405.

10. Foucard, P., Tocqueville, A., Gaumé, M., Labbé, L., Baroiller, J. F., Lejolivet, C., Lepage, S. et Arfeuille, B. (2015). Tour d'horizon du potentiel de développement de l'aquaponie en France : présentation et regard critique sur cette voie de développement alternative pour les productions piscicoles et horticoles. Innovations Agronomiques, $15 \mathrm{p}$. Disponible sur: https://creativecommons.org/licenses/by-ncnd/3.0/fr/. Consulté en ligne le 17 août 2019.

11. Gidinda, Y. (2016). Essai comparatif des trois fertilisants organiques sur la production du Gombo (Abelmoscus esculentus L. Moench) dans les conditions écologiques de Kinshasa/Mont-Amba. Mémoire de Licence, Faculté des Sciences Agronomiques, Université de Kinshasa, R.D Congo, $50 \mathrm{p}$.

12. Heliott, T. (2014). Biodiversité des communautés d'invertébrés benthiques des rivières de la Guadeloupe et responses aux perturbations anthropiques. Thèse de doctorat, Spécialité : Ecologie des systèmes aquatiques, Université Paul Sabatier-Toulouse III, France, 256 p.

13. Kestemont, P., Micha J-.C. et Falter, U. (1989). Les Méthodes de Production d'Alevins de Tilapia nilotica. Project reports ADCP/REP/89/46, PNUD-FAO, Rome, $132 \mathrm{p}$.

14. Kloas, W. (2015). A new concept for aquaponic systems to improve sustainability, increase productivity, and reduce environmental impacts. Aquaculture Environment Interactions, 7 : 179-192.

15. Lazard, J. (2009). La pisciculture des tilapias. Cahiers Agricultures, 18(2): 393-401. 
16. Laviale, R. (2015). Expérimentation et démonstration aquaponique à vocation pédagogique, optimisation pilote régionale en aquaponie. Université Savoie Mont Blanc, France, 73 p.

17. Lusasi, S. W., Makiese, M. P., Kunonga, N. L., Munganga, K. C., Kavumbu, M. S. et Pwema, K. V. (2019b). Proportion de vente des poissons frais locaux et importés dans les marchés de Kinshasa en République Démocratique du Congo (cas des marchés de la Liberté de Masina et Central de Kinshasa). Journal of Applied Biosciences, 141: 14353 - 14363. https://dx.doi.org/10.4314/jab.v141i1.2.

18. Lusasi, S. W., Pwema, K. V., Munganga, K. C., Kavumbu, M. S. et Mutambwe, S. (2019a). Mise au point d'un aliment pour Distichodus maculatus Boulenger, 1898 à base des sous-produits agricole disponibles localement. Afrique SCIENCE, 15(2) : 238 - 248.

19. Masua, T. B., Lusasi, S. W., Munganga, K. C., Wumba, M. P., Kavumbu, M. S. et Pwema K. V. (2020). Inventory of fresh fish marketed in the markets of Kinshasa in the Democratic Republic of Congo (case of the Gambela and Matete markets). International Journal of Applied Research, 6(4): 102-108.

20. McBratney, A., Whelan, B. and Ancev, T. (2005). Futur directions of precision agriculture. Précision Agriculture, 6 : 7-23.

21. MDDELCC (Ministère du Développement Durable, de l'Environnement et de la Lutte contre les Changements Climatiques). (2017). Guide de caractérisation physicochimique de l’état initial du milieu aquatique avant l'implantation d'un projet industriel, Québec. Direction générale du suivi de l'état de l'environnement, ISBN 978-2550-79556-8, 12 p + annexes.

22. Melard, C. et Philippart, J. C. (1981). La production de tilapia de consommation dans les rejets industriels d'eau chaude en Belgique. Cahiers d'Ethologie appliquée, collection Enquêtes et dossiers : 2(1):2, 122 p.

23. Micha, J-.C. (1975). Synthèse des essais de reproduction, d'alevinage et de production chez un silure africain : C. gariepinus. FAO/CPA, Aquaculture in Africa, Ghana CIFA. Pap. Techn, 4(1) : 450-463.

24. MINAGRI (Ministère d'Agriculture/RDC). (2012). Programme National de Sécurité alimentaire (P.N.S.A). Version amendée après l'atelier national du 16 Décembre 2010, 115 p.

25. Munganga, K. C., Kavumbu, M. S., Bipendu, M. N., Kunonga, N. L., Lusasi, S. W. et Pwema, K. V. (2020). Evaluation de la Qualité Écologique de la Rivière Musolo à Kinkole Basée sur les Macroinvertébrés Benthiques (Kinshasa, R.D Congo). European Journal of Scientific Research, 155(2) : 162-176. 
26. Picolo, A., Short, C. et Sommerville, C. (2013). Aquaponie - une solution intelligente basée sur les poissons pour produire de la nourriture en utilisant des ressources limitées et peu d'eau. SMARTFISH, $4 \mathrm{p}$.

27. Pouomogne, V. (1998). Pisciculture en milieu tropical Africain : Comment produire du poisson à coût modéré. Coopération française, Centre d'excellence pour la production, l'innovation et le développement. Presse Universitaire d'Afrique, Yaoundé, Cameroun, $236 \mathrm{p}$.

28. Saidani, I. (2016). Essai d'installation d'un système aquaponique dans une ferme agricole : Etude Technique, Mise en place et premiers résultats d'exploitation. Projet de fin d'études du cycle d'ingénieur, Département d'Agronomie et Biotechnologie Végétale, Institut National Agronomique de Tunisie, $94 \mathrm{p}$.

29. Stalport, B. (2017). Modélisation et développement d'un système aquaponique avec surveillance métrologique pour l'étude du cycle de l'azote. Mémoire de Master en bioingénieur : sciences et technologies de l'environnement, à finalité spécialisée, ULG, Agro-Bio Tech (GxABT), 103 p. Disponible sur : http://matheo.ulg.ac.be.

30. Tollens, E. (2015). Les parcs agro-industriels et l'agriculture familiale. Les défis du secteur agricole en République Démocratique du Congo, $12 \mathrm{p}$. 